\title{
Laminar momentum jets in a stratified fluid
}

\author{
By E. J. LIST \\ W.M. Keck Laboratory of Hydraulics and Water Resources \\ California Institute of Technology
}

(Received 27 February 1970 and in revised form 24 September 1970)

Solutions are presented for creeping flows induced by two- and three-dimensional horizontal and vertical momentum jets in a linearly stratified unbounded diffusive viscous fluid. These linear problems are solved by replacing the momentum jet by a body force singularity represented by delta functions and solving the partial differential equations of motion by use of multi-dimensional Fourier transforms. The integral representations for the physical variables are evaluated by a combination of residue theory and numerical integration.

The solutions for vertical jets show the jet to be trapped within a layer of finite thickness and systems of rotors to be induced. The horizontal twodimensional jet solution shows return flows above and below the jet and a pair of rotors. The three-dimensional horizontal jet has no return flow at finite distance and the diffusive contribution is found to be almost negligible in most situations, the primary character of the horizontal flows being given by the non-diffusive solution. Stokes's paradox is found to be non-existent in a densitystratified fluid.

\section{Introduction}

The need for an understanding of the behaviour of jets in density-stratified fluids has become necessary in the study of ocean outfalls and reservoir mixing systems. Buoyant turbulent jets have received a great deal of attention since the paper by Morton, Taylor \& Turner (1956) but there is apparently little knowledge of the growth rate of neutrally buoyant and horizontal momentum jets in stratified fluids. These are extraordinarily difficult problems due to the differential growth rates in the vertical and horizontal directions and it was felt that in the same way that laminar jets in homogeneous fluids cast some light on the behaviour of turbulent jets, a study of laminar jets in stratified fluids might well be fruitful.

Flows generated by sources of momentum in viscous fluids have been studied for over 100 years. The portion of Stokes's solution for the slow motion of a sphere can be interpreted as the flow generated by a weak momentum jet, the so-called stokeslet solutions of the Navier-Stokes equations (Hancock 1953). An exact solution for a horizontal three-dimensional momentum jet was discussed by Landau (1944) following Slezkin's (1934) integral of the Navier-Stokes equation and also by Squire,(1951). This solution reduces to Schlichting's (1933) boundary-layer solution for strong jets and to the stokeslet solution for weak 
jets. A corresponding integral for two-dimensional momentum jets apparently has not to date been found.

Little work has been done on the analogous problems in stratified fluids. Long (1959) neglected inertial forces and made the boundary-layer assumption to find a similarity solution for the flow induced by a body moving at constant speed in a stratified fluid and later, (Long 1962) extended this boundary-layer solution to include diffusive effects. Janowitz (1968) has recently found a solution for the flow induced by a small two-dimensional obstacle moving horizontally in a linearly stratified, viscous, non-diffusive fluid. The Janowitz solution is in effect the two-dimensional oseenlet solution for a linearly stratified, viscous, non-diffusive fluid.

A full non-linear analysis or even a non-linear boundary-layer analysis for momentum jets in stratified fluids is not easy and as will be seen later is not practicable for vertical jets. For horizontal jets a boundary-layer type solution is possible for the two-dimensional jet, as Long (1959) has shown, but at first sight such an analysis for a three-dimensional jet appears difficult. At this juncture not even Stokes's problem has been solved in a stratified fluid and in the same way that a study of this problem in viscous fluids leads to an Oseen type analysis and later a boundary-layer analysis it was felt that a study of the stokeslet solutions may well be worthwhile, especially for the three-dimensional jets. Thus, this paper is an attempt to find the corresponding stokeslet solutions for a linearly stratified viscous diffusive fluid. The problem is made more interesting than the corresponding viscous flow problems by the fact that the solutions depend upon the relative orientation of the stokeslet and the gradient of the density field.

The problem is solved by introducing the momentum jets as body force singularities in the linearized equations of motion. Multi-dimensional Fourier transforms are then used to convert the differential equations to algebraic equations which are easily solved. The Fourier inversion is carried out by doing one integration directly (necessary in three-dimensions) using residue theory on another and finally employing numerical integration. The stream function and density anomaly are computed and contours plotted to depict the flow fields. The flow fields are found to be symmetric in both the horizontal and the vertical co-ordinates and there are systems of rotors and reverse flows induced except for the three-dimensional horizontal jet, which has no reverse flow at finite distance. All velocities are found to vanish at infinity so that Stokes's paradox does not occur in stratified fluids.

\section{Statement of the problem}

We consider an unbounded Newtonian diffusive density-stratified fluid for which the equations of motion are the following: the equation of mass conservation,

$$
\nabla \cdot(\rho \mathbf{u})=D \nabla^{2} C,
$$

where $\rho$ is the fluid density, $\mathbf{u}$ the velocity vector with components $u, v, w$ and $C$ 
is the concentration of a tracer causing the density variation, and $D$ the molecular diffusivity of the tracer; the equation of conservation of tracer,

$$
\nabla .(C \mathbf{u})=D \nabla^{2} C
$$

and the equation of momentum conservation

$$
\rho(\mathbf{u} . \nabla) \mathbf{u}=-\nabla p^{\prime}+\mu \nabla^{\mathbf{2}} \mathbf{u}-\rho g \mathbf{j}+\mathbf{X}
$$

where $p^{\prime}$ is the fluid pressure, $\mu$ is the dynamic viscosity and $\mathbf{X}$ the body force per unit volume, $\mathbf{j}$ is a unit vector in the upward vertical direction. For small density changes the density and tracer concentration can be related by an equation of state,

$$
\rho-\rho_{0}=\beta\left(C-C_{0}\right)
$$

where $\rho_{0}$ is a conveniently chosen reference density and $C_{0}$ its associated concentration, $\left(\beta \simeq 0.67\right.$ for a sodium chloride solution at $\left.20^{\circ} \mathrm{C}\right)$.

Equations (1) and (2) are only valid in regions containing no sources or sinks and are suitable for use here where singularities of mass or concentration are excluded. These two equations can be rewritten in the form

$$
\begin{gathered}
\nabla . \mathbf{u}=\alpha D \nabla^{2}\left(\rho / \rho_{0}\right), \\
\mathbf{u} . \nabla \rho=D\left(\mathbf{l}-\alpha \beta C / \rho_{0}\right) \nabla^{2} \rho,
\end{gathered}
$$

where $\alpha=\rho_{0}(1-\beta) / \beta\left(\rho_{0}-\beta C_{0}\right) \simeq 0.5$ for a $2 \%$ by weight sodium chloride solution in water at $20^{\circ} \mathrm{C}$. In the following we ignore the right-hand side of (5) and assume $\alpha \beta C / \rho_{0} \ll 1$ in $(6)$. We consider the case now when the density distribution can be written

$$
\rho / \rho_{0}=1-\epsilon y+s^{\prime}(x, y, z),
$$

and the body force vector has the form

$$
\mathbf{X}=(X \mathbf{i}+X \mathbf{j}+Z \mathbf{k}) \delta(\mathbf{x})
$$

where a system of right-handed Cartesian co-ordinates has been chosen with the $y$ co-ordinate vertical. The basic density profile is linear with a constant slope $\epsilon$ and $s^{\prime}(x, y, z)$ is the density anomaly arising from the presence of the body force singularity with components $X, Y$, and $Z$ and denoted by the Dirac delta functions, $\delta(\mathbf{x})$. The body force singularity may be considered to be a point source of momentum located at the origin, i.e. a momentum jet. Under these circumstances (6) and (3) take the form

and

$$
\text { u. } \nabla s^{\prime}-\epsilon v=D \nabla^{2} s^{\prime},
$$

where

$$
\begin{aligned}
\rho(\mathbf{u} . \nabla) \mathbf{u} & =-\nabla P-\rho_{0} g s^{\prime} \mathbf{j}+\mathbf{X}+\mu \nabla^{2} \mathbf{u} \\
P & =p^{\prime}+\rho_{0} g\left(y-\frac{1}{2} \epsilon y^{2}\right)
\end{aligned}
$$

(As Janowitz (1968) has stated, the basic linear density profile as specified by (7) is certainly not possible over an extreme range of $y$ values, but is a good approximation over a large enough distance that solutions can be obtained representing, say, the behaviour in the centre of a hyperbolic tangent density profile.)

An investigation of the validity of a linearization of these equations of motion can be carried out in non-dimensional variables chosen so that the linear terms are all of an equal order of magnitude. 
Consider the two-dimensional case first and suppose that $F$ is a measure of the source strength per unit length. Choosing a characteristic length

$$
l=\left(\mu D / \rho_{0} g \epsilon\right)^{\frac{1}{4}},
$$

a characteristic velocity

$$
\begin{gathered}
U_{0}=F / \mu, \\
s=\left(\rho_{0} g D \mu / \epsilon F^{2}\right)^{\frac{1}{2}} s^{\prime},
\end{gathered}
$$$$
\text { and letting }
$$

implies the equations of motion take the form, in dimensionless variables,

$$
\begin{gathered}
Q \mathbf{u} \cdot \nabla s-v=\nabla^{2} s \\
Q(D / v)(\mathbf{u} \cdot \nabla) \mathbf{u}=-\nabla p-s \mathbf{j}+\mathbf{F}_{(n)} \delta(\mathbf{x})+\nabla^{2} \mathbf{u}
\end{gathered}
$$

where

$$
\begin{gathered}
Q=(F / \mu D)\left(\mu D / \rho_{0} g \epsilon\right)^{\frac{1}{4}}, \\
p=P l / F,
\end{gathered}
$$

and

$$
\mathbf{F}_{(2)}=(X \mathbf{i}+Y \mathbf{j}) F^{-1} \text {. }
$$

In three dimensions we choose the same length scale $l$, a characteristic velocity $F / \mu l$ and write

$$
s=(\mu D / \epsilon F l) s^{\prime},
$$

to get forms similar to (13) and (14) except that now

$$
\begin{gathered}
Q=F / \mu D, \\
p=P l^{2} / F, \\
\mathbf{F}_{(3)}=(X \mathbf{i}+Y \mathbf{j}+Z \mathbf{k}) F^{-1} .
\end{gathered}
$$

Since for most liquids $D / \nu$ is not large, the criterion for the linear solutions to be appropriate is that $Q \ll 1$, the appropriate $Q$ being chosen in each case.

The linearized equations (13) and (14) with the appropriately chosen $\mathbf{F}_{(n)}$ are most easily solved by use of the multi-dimensional Fourier transform

$$
\Phi(\boldsymbol{\kappa})=(2 \pi)^{-\frac{1}{2} n} \int_{-\infty}^{\infty} \phi(\mathbf{x}) \exp (i \mathbf{\kappa} \cdot \mathbf{x}) d \mathbf{x}
$$

where $n$ takes the value 2 or 3 depending upon whether $\mathrm{x}$ and $\boldsymbol{\kappa}$ are two- or three-dimensional vectors. Application of this Fourier transform leads to algebraic equations for the transformed variables $\mathbf{U}=(U, V, W), P$, and $S$ involving only the constant force vector $\mathbf{F}=\mathbf{F}_{(n)} /(2 \pi)^{\frac{1}{2} n}$. The solutions to these algebraic equations are

$$
\begin{aligned}
& \kappa^{2} \mathbf{U}=\mathbf{F}+\left\{\mathbf{j}\left[(\boldsymbol{\kappa} \cdot \mathbf{j})(\boldsymbol{\kappa} . \mathbf{F})-\kappa^{2}(\mathbf{j} . \mathbf{F})\right]\right. \\
& \left.+\kappa\left[(\boldsymbol{\kappa} . \mathbf{j})(\mathbf{j} . \mathbf{F})-\left(\kappa^{4}+1\right)(\boldsymbol{\kappa} . \mathbf{F})\right]\right\} /\left[\kappa^{2}\left(\kappa^{4}+1\right)-(\mathbf{j} \cdot \boldsymbol{\kappa})^{2}\right], \\
& P=-i\left[(\boldsymbol{\kappa} . \mathbf{j})(\mathbf{j} . \mathbf{F})-\left(\kappa^{4}+1\right)(\boldsymbol{\kappa} . \mathbf{F})\right] /\left[\kappa^{2}\left(\kappa^{4}+1\right)-(\mathbf{j} \cdot \boldsymbol{\kappa})^{2}\right] \text {, } \\
& S=-\left[(\boldsymbol{\kappa} . \mathbf{j})(\kappa . \mathbf{F})-\kappa^{2}(\mathbf{j} \cdot \mathbf{F})\right] /\left[\kappa^{2}\left(\kappa^{4}+1\right)-(\mathbf{j} \cdot \boldsymbol{\kappa})^{2}\right] .
\end{aligned}
$$

We now consider some particular types of force vectors corresponding to various momentum jets, when $\mathbf{F}$ takes the appropriate value. 


\section{Two-dimensional jets}

For a two-dimensional jet the force function $F$ takes the form given by (17) and the results are summarized in table 1.

$\begin{array}{ccc}\text { Transform } & \text { Coefficient of } \bar{X} / 2 \pi & \text { Coefficient of } \bar{Y} / 2 \pi \\ U & \kappa_{2}^{2} \kappa^{2} / d & -\kappa_{1} \kappa_{2} \kappa^{2} / d \\ V & -\kappa_{1} \kappa_{2} \kappa^{2} / d & \kappa_{1}^{2} \kappa^{2} / d \\ P & i \kappa_{2}\left(\kappa^{4}+1\right) / d & i \kappa_{2} \kappa^{4} / d \\ S & -\kappa_{1} \kappa_{2} / d & \kappa_{1}^{2} / d \\ \Psi & +i \kappa_{2} \kappa^{2} / d & -i \kappa_{1} \kappa^{2} / d\end{array}$

TABLE 1. Fourier transforms for a two-dimensional jet, $d=\kappa^{6}+\kappa_{1}^{2}, \bar{X}=X F^{-1}, \bar{Y}=Y F^{-1}$

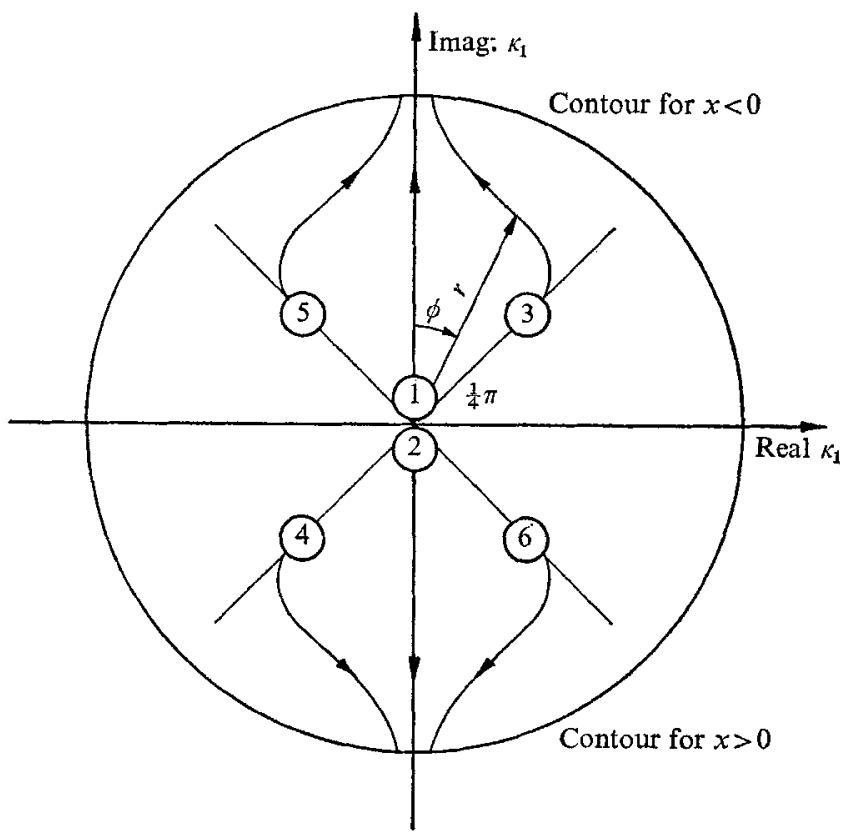

(a)

FIGURE 1. Location of the zeros of $\kappa^{6}+\kappa_{1}^{2}$ given by equations (25) and plotted in the $\kappa_{1}$ plane when $\kappa_{2}=0$. The arrows and lines give the trajectories of the zeros as $\kappa_{2}$ increases from zero.

The physical variables, including the stream function $\Psi$, can be found using the Fourier inversion integral

$$
\phi(\mathbf{x})=(2 \pi)^{-\frac{1}{2} n} \int_{-\infty}^{\infty} \Phi(\kappa) \exp (-i \kappa . \mathbf{x}) d \kappa
$$

where in this case $n=2$ and the integral is over two dimensions. All the transforms have the same poles and they are located at the zeros of $\kappa^{6}+\kappa_{1}^{2}$, which are

$$
\left.\begin{array}{l}
\kappa_{(1),(2)}=\left[\left(\frac{2}{3}\right)^{\frac{1}{2}} \sinh \frac{1}{3} \theta\right]^{\frac{3}{2}} \exp \left( \pm \frac{1}{2} i \pi\right)=\rho \exp \left( \pm \frac{1}{2} i \pi\right), \\
\kappa_{(3),(5)}=r \exp \left[i\left(\frac{1}{2} \pi \pm \phi\right)\right], \\
\kappa_{(4),(6)}=r \exp \left[i\left(\frac{3}{2} \pi \pm \phi\right)\right],
\end{array}\right\}
$$


and are located as shown in figure 1 and where

$$
\begin{aligned}
\phi & =\frac{1}{2} \arctan \left(2 \cosh \frac{1}{3} \theta /\left(2 \kappa_{2}^{2}+\rho^{2}\right)\right), \\
\theta & =\operatorname{arcsinh}\left(3 \frac{3}{2} \frac{1}{2} \kappa_{2}^{2}\right), \\
r & =\left(\rho^{\frac{4}{3}}+1\right)^{\frac{3}{2}} .
\end{aligned}
$$

The integrals can now be evaluated in each half-plane corresponding to the sign of $x$ by a straightforward but tedious application of residue theory. The results for variables of interest are found in a form exemplified by (27)

$$
f(x, y)=\frac{A}{2 \pi} \int_{0}^{\infty} \frac{K(y, t)}{3 \rho^{\frac{4}{3}}+1}\{L(t) \exp [-\rho|x|]-M(x, t) \exp [-r|x| \cos \phi]\} d t
$$

where, for example, for the two-dimensional horizontal jet stream function

$$
\begin{aligned}
& f(x, y)=\Psi(x, y), \quad K(y, t)=t \sin y t, \quad L(t)=\rho^{-\frac{1}{3}}, \quad A=\bar{X}, \quad t=\kappa_{2}, \\
& M(x, t)=\left[3^{-\frac{1}{2}} \sinh \left(\frac{2}{3} \theta\right) \cos (\phi+r|x| \sin \phi)\right. \\
& \left.-\cosh \left(\frac{2}{3} \theta\right) \sin (\phi+r|x| \sin \phi)\right] / r \cosh \left(\frac{1}{3} \theta\right) .
\end{aligned}
$$

\section{Results for two-dimensional jets}

Solutions for two physical variables were evaluated by numerical integration and the results are presented in terms of contours of stream function and density anomaly.

The integrals were evaluated over the range $x=0(0 \cdot 5) 20, y=0(0 \cdot 5) 15$, using a double precision 16-point Gaussian integration formula with a period $\pi / y$ and summing successive contributions until less than $10^{-8}$. A contouring subroutine was used to plot the curves of constant stream function and constant density anomaly. For $x=0$, the integral for $\Psi$ is very slowly converging and a method given by Longman (1956) for the numerical evaluation of oscillatory integrals was used. Total computer time to cover the $x, y$ field as specified above was approximately 90 seconds.

For the horizontal jet the most striking result is the formation of rotors above and below the jet. This is shown in the detailed structure in the neighbourhood of the jet presented in figure 2. This is in keeping with the experiments by Long (1959) where a series of rotors is seen above a moving ridge at the base of a density stratified fluid. The experiments show the rotor to be pushed to the rear of the ridge and to be somewhat distorted in shape from that shown here. However, the basic phenomenon is present even in the creeping flow presented here. There appears to be only one rotor above the jet in this linear solution case. This is the characteristic feature of the density stratified flow, for a rotor such as this does not appear at a finite distance from the jet in a non-stratified viscous. fluid. It is apparent that the effect of the vertical density gradient has been to confine the return flow to a finite layer about the source point thus inducing the rotors near the jet.

The density anomaly for the horizontal jet is given in figure 3 . The effect of the jet is to introduce a positive density anomaly above the jet downstream and a negative anomaly below the jet downstream; the signs are reversed upstream. 


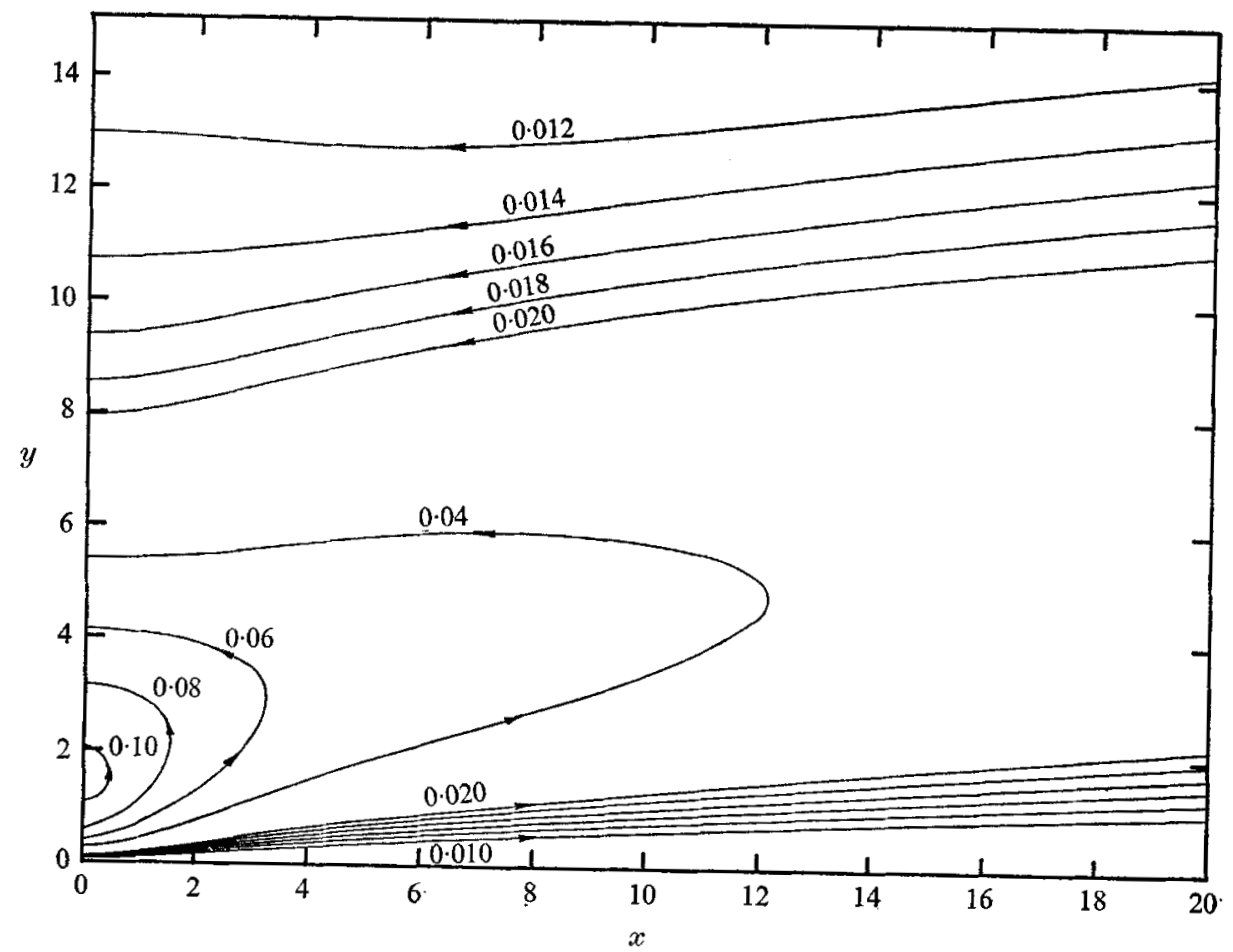

Figure 2. Contours of non-dimensional stream function $\Psi$ for a two-dimensional horizontal jet, axes are non-dimensional co-ordinates $x$ and $y$.

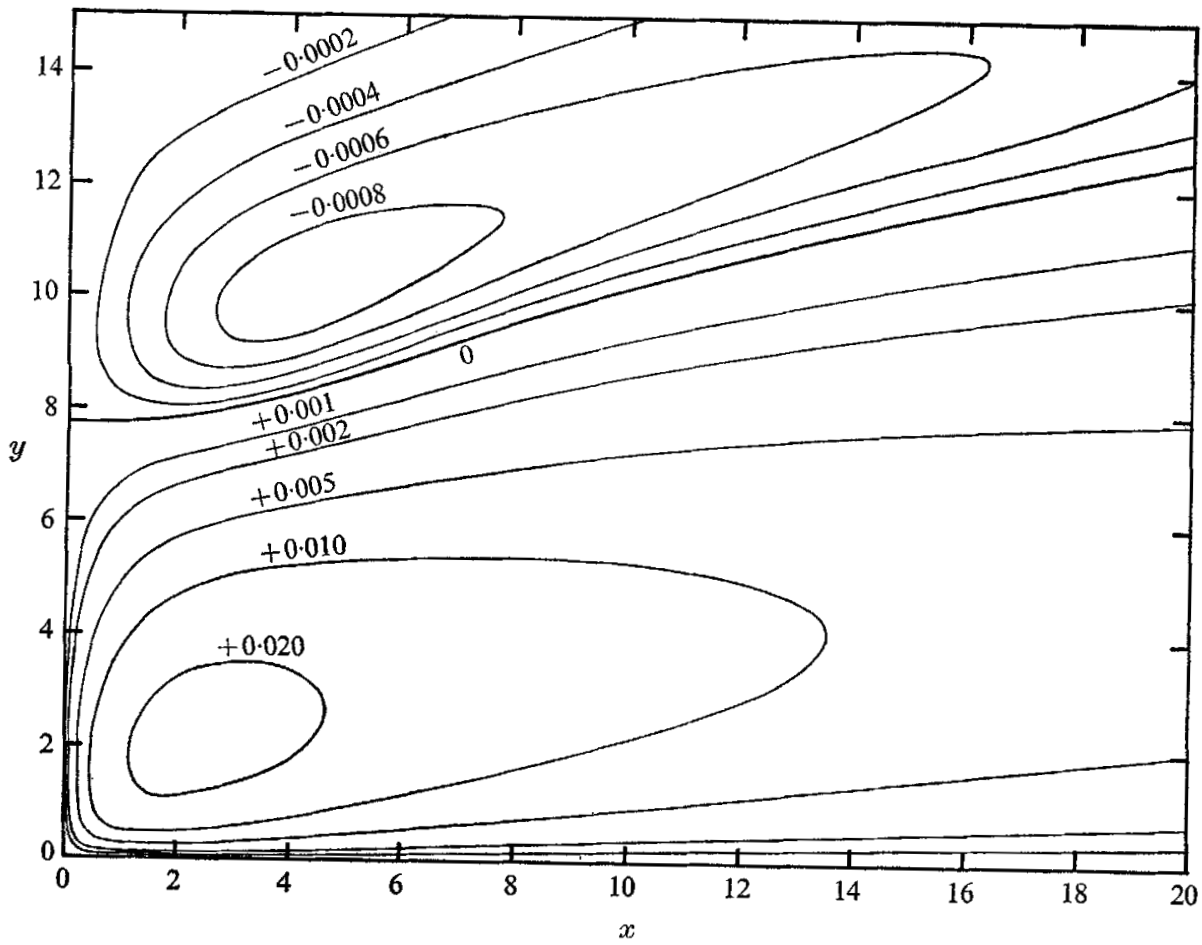

FIGURE 3. Contours of density anomaly $s$ for a two-dimensional horizontal jet, axes are non-dimensional co-ordinates $x$ and $y$. 


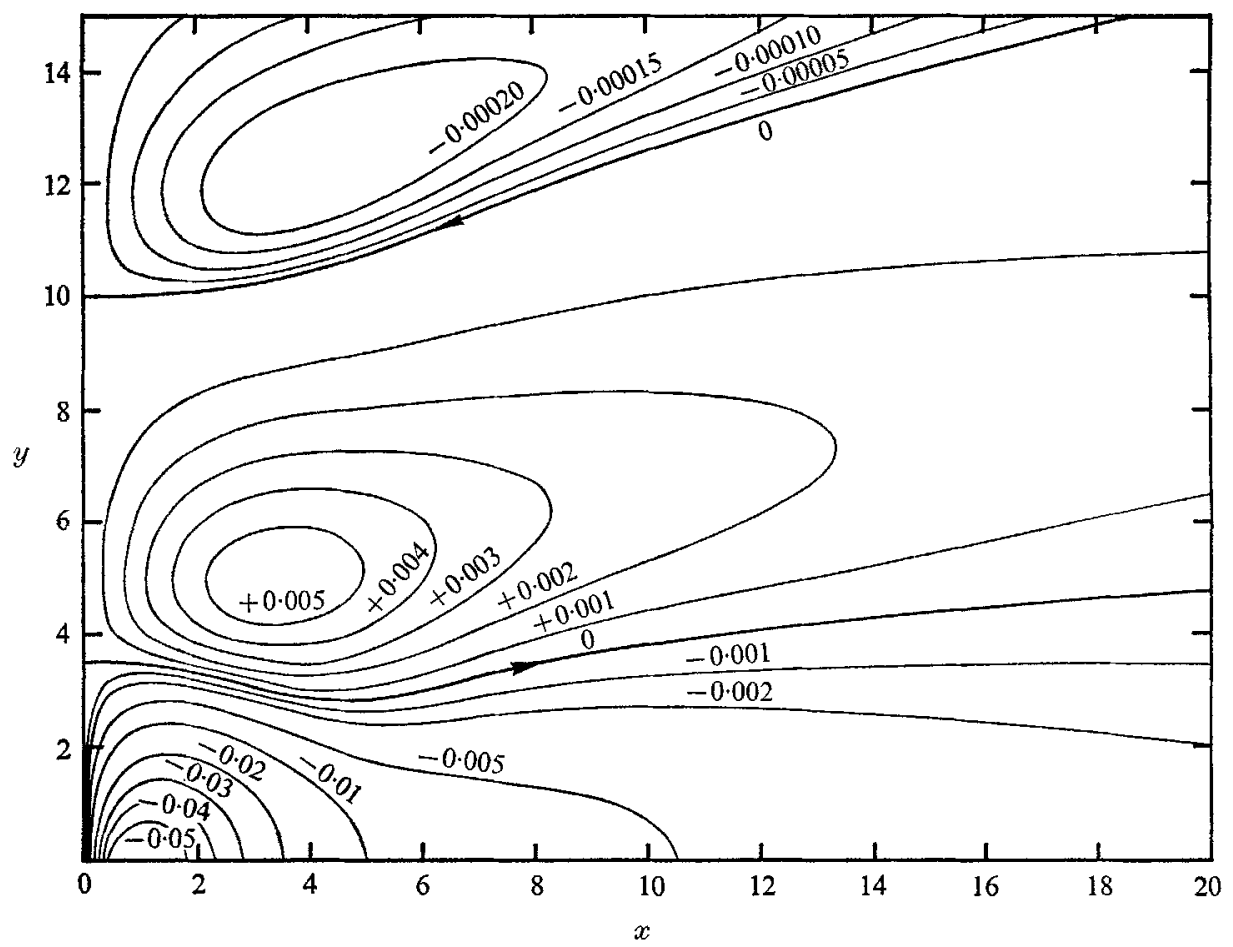

FIGURE 4. Contours of non-dimensional stream function $\Psi$ for a two-dimensional vertical jet. axes are non-dimensional co-ordinates $x$ and $y$.

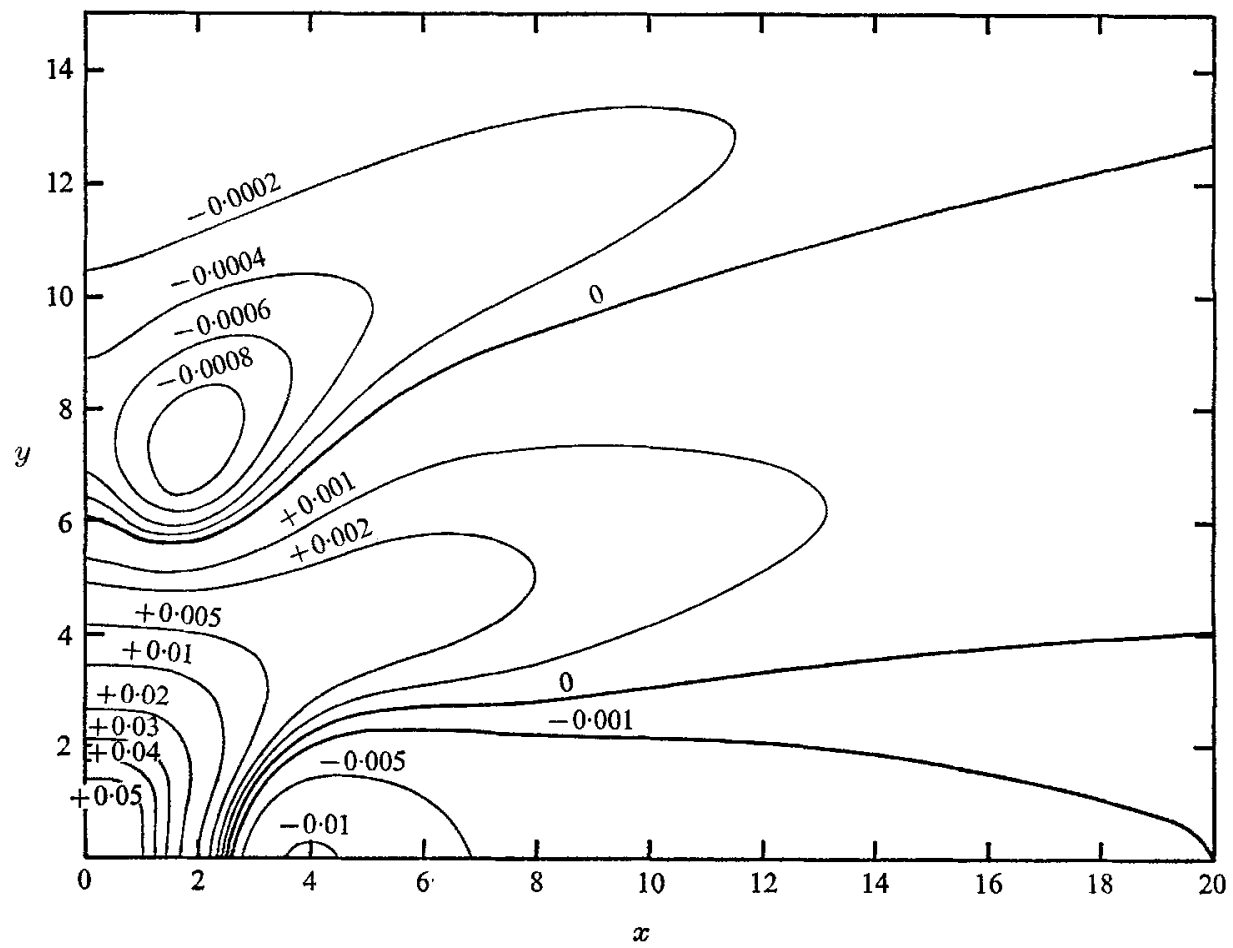

FigURE 5. Contours of density anomaly $s$ for a two-dimensional vertical jet, axes are nondimensional co-ordinates $x$ and $y$. 
We can therefore expect the downstream jet to be significantly more unstable than the upstream jet. This result should be valid for the non-linear jet also.

Solutions for the vertical two-dimensional momentum jet are given in figures 4 and 5 . Figure 4 shows the stream function contours for a vertical jet at the origin and in this case there is a rotor on each side of the jet, clockwise on the right and counterclockwise on the left. The fluid has a limited height of rise and two further rotors of opposite sign located above these. The height of rise is independent of the strength of the jet and the total thickness of the primary layer will, of course, be twice that shown. The anomaly shows the characteristic plume-like structure close to the momentum source but departs markedly from this away from the source. The vertical momentum flux apparently supports the positive density anomaly until such a point that the total negative buoyancy overcomes it whereupon the fluid falls back down. There appears to be a stability problem on the lateral fringes of the jet where it is seen a wedge of lighter fluid is overlaid by heavier fluid.

A full scale stability analysis of the two-dimensional problems above would be difficult and is beyond the scope of this work. However, we can define a local perturbation Rayleigh number at locations where an adverse perturbation density gradient exists. The depth of a layer containing such a gradient appears to be bounded (see figures 3 and 5 ), and will be proportional to $\left(\mu D / \rho_{0} g \epsilon\right)^{\frac{1}{4}}$. The magnitude of any adverse gradient is equal to

$$
\left|\frac{\partial s}{\partial y}\right| \cdot\left[\frac{\epsilon F^{2}}{\rho_{0} g D \mu}\right]^{\frac{1}{2}} \cdot\left(\frac{\rho_{0} g \epsilon}{\mu D}\right)^{\frac{1}{4}},
$$

and it is apparent that $|\partial s| \partial y \mid$ is also bounded (see figures 3 and 5 ), so the local Rayleigh number will be proportional to

$$
Q=\frac{F}{\mu D} \cdot\left[\frac{\mu D}{\rho_{0} g \epsilon}\right]^{\frac{1}{4}}
$$

Thus, the convective stability parameter is seen to be exactly the parameter we found controlling the applicability of the linear solution. If $Q \ll 1$ then the linear solutions found are not only valid but would appear to be at least convectively stable also. Obviously, other parameters will also enter the overall jet stability problem.

These solutions are for linear equations and can be superimposed in any way. Thus solutions for jets at any angle are possible by taking an appropriate linear combination of the vertical and horizontal solutions.

\section{Three-dimensional jets}

In this case the force function $F$ takes the form given by (21). The corresponding values of the transformed velocity vector, pressure field and density anomaly are given in table 2.

It will be noted that the transforms for a vertical jet are symmetrical in $\kappa_{1}$ and $\kappa_{3}$ as we would expect for an axisymmetrical problem, but this is not true for the horizontal jet. It is this latter problem which is of major interest since it is a 
truly three-dimensional problem. Fortunately, however, we note that the denominators of the transformed variables are symmetric in $\kappa_{1}$ and $\kappa_{3}$ and this symmetry can be exploited in the Fourier inversion integral to facilitate the integration.

$\begin{array}{ccc}\text { Transform } & \text { Coefficient } \bar{X} /(2 \pi)^{\frac{3}{2}} & \text { Coefficient } \bar{Y} /(2 \pi)^{\frac{3}{2}} \\ \kappa^{2} \mathbf{U} & {\left[\mathbf{i}\left(\kappa^{4}\left(\kappa_{2}^{2}+\kappa_{3}^{2}\right)+\kappa_{3}^{2}\right)-\mathbf{j} \kappa_{1} \kappa_{2} \kappa^{4}\right.} & \left(\kappa^{4} \mathbf{j}-\kappa_{2} \kappa^{2} \mathbf{k}\right) / d \\ P & \left.-\mathbf{k} \kappa_{1} \kappa_{3}\left(\kappa^{4}+1\right)\right] / d & \\ P & i \kappa_{1}\left(1+\kappa^{4}\right) / d & i \kappa_{2} \kappa^{4} / d \\ S & -\kappa_{1} \kappa_{2} / d & \left(\kappa^{2}-\kappa_{2}^{2}\right) / d\end{array}$

TABLE 2. Fourier transforms for a three-dimensional jet, $d=\kappa^{6}+\kappa_{1}^{2}+\kappa_{3}^{2}, \bar{X}=X F^{-1}$,

$$
\bar{Y}=Y F^{-1} \text {. }
$$

Let

$$
\begin{array}{ll}
x=\sigma \cos \lambda, & \kappa_{1}=t \cos \omega, \\
z=\sigma \sin \lambda, & \kappa_{3}=t \sin \omega,
\end{array}
$$

then the inversion integral takes the form

$$
\begin{array}{r}
\phi(\mathbf{x})=(2 \pi)^{-3} \int_{0}^{2 \pi} \int_{-\infty}^{\infty} \int_{0}^{\infty} \frac{\exp \left(-i \kappa_{2} y\right) \exp [-i t \sigma \cos (\lambda-\omega)] \cos m \omega}{\left(\kappa_{2}^{2}+t^{2}\right)^{3}+t^{2}} \\
\times \Phi\left(\kappa_{2}, t^{2}\right) t d t d \kappa_{2} d \omega .
\end{array}
$$

There are two things to note about this integral. First, the $\omega$ integration can be carried out directly when $m$ is an integer, and second, the denominator is now in the same form as for the two-dimensional jet case and the zeros are therefore already known. However, in this particular case it is more convenient to find the zeros in terms of the other variable and thereby avoid Kelvin functions in favour of Bessel functions. The zeros are

$$
\left.\begin{array}{l}
\kappa_{(1),(2)}=\left(t^{2}+t^{\frac{2}{3}}\right)^{\frac{1}{2}} \exp \left[ \pm \frac{1}{2} i \pi\right]=\rho \exp \left[ \pm \frac{1}{2} i \pi\right], \\
\kappa_{(3),(4)}=R \exp [ \pm i \phi], \\
\kappa_{(5),(6)}=R \exp [i(\pi \pm \phi)],
\end{array}\right\}
$$

where

$$
R=\left(t^{4}-t^{\frac{8}{3}}+t^{\frac{4}{3}}\right)^{\frac{1}{4}}
$$

$$
\phi=\left\{\begin{array}{l}
\frac{1}{2} \arctan \left(3^{\frac{1}{2}} /\left(1-2 t^{\frac{4}{3}}\right)\right) \quad\left(t^{\frac{4}{3}}<\frac{1}{2}\right), \\
\frac{1}{2}\left[\pi-\arctan \left(3^{\frac{1}{2}} /\left(2 t^{\frac{4}{3}}-1\right)\right)\right] \quad\left(t^{\frac{4}{3}}>\frac{1}{2}\right) .
\end{array}\right.
$$

The integrals are evaluated using residue theory as before and the solutions have the form

$$
f(\sigma, y)=\int_{0}^{\infty} P(\sigma, t)[A(y, t) \exp (-|y| R \sin \phi)+B(t) \exp (-\rho|y|)] d t
$$

where, for example, for the vertical axisymmetric jet stream function,

$$
\begin{gathered}
f(\sigma, y)=\Psi(\sigma, y), \quad P(\sigma, t)=\sigma t J_{1}(\sigma t) / 12 \pi, \quad B(t)=-t^{\frac{2}{3}} \rho, \\
A(y, t)=2 t^{\frac{7}{3}} \cos \left(\phi-|y| R \cos \phi-\frac{1}{6} \pi\right) / R .
\end{gathered}
$$


For the horizontal three-dimensional jet the solutions take the form $(\bar{X}=1)$ :

$$
\begin{aligned}
u(x, y, z) & =(8 \pi)^{-1}\left\{r^{-1}+(r-|y|)[r(r+|y|)]^{-1} \cos 2 \lambda+f_{1}(\sigma, y)\right. \\
v(x, y, z) & =(8 \pi)^{-1} \cos \lambda f_{3}(\sigma, y) \\
w(x, y, z) & =(8 \pi)^{-1} \sin 2 \lambda\left\{(r-|y|)[r(r+|y|)]^{-1}-f_{2}(\sigma, y) \cos 2 \lambda\right\} \\
s(x, y, z) & =(8 \pi)^{-1} \cos \lambda f_{4}(\sigma, y)
\end{aligned}
$$

where $f_{1}, f_{2}, f_{3}, f_{4}$ are represented by integrals of the form (30) and

$$
r=\left(x^{2}+y^{2}+z^{2}\right)^{\frac{1}{2}}, \quad \sigma=\left(x^{2}+z^{2}\right)^{\frac{1}{2}}, \quad \lambda=\arctan (z / x) .
$$

\section{Results for three-dimensional jets}

The overall structure of stream function and density anomaly for the vertical three-dimensional jet, when seen in section, is very similar to the two-dimensional vertical jet and hence detailed results are not given here. Stagnation points are located at $y= \pm 3 \cdot 786, \pm 9 \cdot 404$ and $\pm 15 \cdot 807$ and there will probably be others outside this range but the velocity values are so small that within the third layer the fluid is virtually stagnant. The rotor induced by the jet is now of course a toroidal one. The density anomaly contours in section are very similar to figure 5 .

The results for the three-dimensional horizontal jet are quite interesting. Consider first of all the explicit part of the solution,

$$
\begin{aligned}
& u=\frac{1}{8 \pi}\left\{\frac{1}{r}+\frac{1}{r}\left[\frac{r-|y|}{r+|y|}\right] \cos 2 \lambda\right\}, \\
& w=\frac{1}{8 \pi} \frac{1}{r}\left[\frac{r-|y|}{r+|y|}\right] \sin 2 \lambda .
\end{aligned}
$$

It may be shown that along with the conditions $v=0, s=0$, these terms are the solution for a horizontal stokeslet in a non-diffusive density-stratified fluid. There is a pressure singularity on the plane $y=0$ which with the viscous terms supplies all the momentum transportfor the jet. However, this pressuresingularity in the $y$-momentum equation must be balanced by a dipole singularity in $s$ on the plane $y=0$, and it is this singularity which provides the inhomogeneous term to generate the solutions $f_{1}, f_{2}, f_{3}$ and $f_{4}$. These latter terms are therefore a solution to the diffusive part of the equations. (This is best seen in cylindrical polar co-ordinates but will not be shown here.) Thus all the vertical motion in the jet is a consequence of the diffusive term in the equations of motion.

It can also be shown that the asymptotic forms of the $f$ functions are selfsimilar as $\sigma$ becomes large, and in fact,

$$
\left.\begin{array}{ll}
f_{1} \sim \sigma^{-\frac{5}{3}} g_{1}(\zeta), & f_{2} \sim \sigma^{-\frac{5}{3}} g_{2}(\zeta), \\
f_{3} \sim \sigma^{-\frac{7}{3}} g_{3}(\zeta), & f_{4} \sim \sigma^{-\frac{5}{3}} g_{4}(\zeta),
\end{array}\right\}
$$

where $\zeta=|y| / \sigma^{\frac{1}{3}}$; the $g$ functions are plotted in figure 6 . 
The relative rates of decay of the diffusive and non-diffusive contributions to the solutions can now be assessed. When $\zeta$ is large we can ignore the diffusive contributions since they may be shown to decay exponentially with large $\zeta$. Hence, for large $y$ at fixed $x$ and $z$

$$
u \sim(8 \pi|y|)^{-1} \quad(y \gg x, z) .
$$

Alternatively, for small $\zeta, g_{1}(\zeta)$ and $g_{2}(\zeta)$ approach their constant values and the asymptotic behaviour of the horizontal velocities becomes dependent upon whether it is $x$ or $z$ which becomes large. If $x$ is large then $\cos 2 \lambda \simeq 1$ and hence

whereas if $z$ is large,

$$
u \sim(4 \pi|x|)^{-1}+O\left(x^{-\frac{5}{3}}\right) \quad(x \gg z, y),
$$

$$
u \sim\left(g_{1}(0)+g_{2}(0)\right) /\left(8 \pi z^{\frac{5}{3}}\right) \quad(z \gg x, y) .
$$

It is apparent therefore that the diffusive contribution to the velocity distribution can be ignored except on the lateral fringes of the jet. The second result above, namely $u \sim(4 \pi|x|)^{-1}$, is exactly the same as for a stokeslet in a homogeneous fluid.

It should also be noted that the vertical velocity vanishes exponentially as $|y|$ becomes large but is of $O\left(\sigma^{-\frac{7}{3}}\right)$ for large $\sigma$ so that it is always at least an order of magnitude less than the lateral horizontal velocity. This vertical velocity is also found to be negative just above the horizontal plane (figure $6(c)$ ) indicating an entrainment flow, whereas the lateral velocity $w$ is seen to be always positive for $x>0, z>0$, indicating no lateral entrainment on the plane $y=0$.

The diffusive part of the solution for the horizontal velocity $u$ does contain negative portions (figure 6) but these are never large enough to overcome the non-diffusive part of the solution; consequently there is no reverse flow at finite distance for the three-dimensional horizontal jet.

This basic difference in behaviour of two- and three-dimensional jets has a simple physical explanation. In the three-dimensional jet a return flow at infinity is possible, just as for a jet in a homogeneous fluid, for the fluid pumped into $x>0$ can return to $x<0$ on the plane $y=0$ without doing work against the density gradient. Thus vertical entrainment into the jet is possible since there is a mechanism for the return flow at infinite distance. In a two-dimensional jet this is not the case, any fluid pumped into $x>0$ cannot return on the plane $y=0$ and also cannot return at infinite distance vertically because of the constraining vertical density gradient, it therefore returns as a reverse flow just above and just below the primary jet. 'This explanation of reverse flows in twodimensional jets and vertical entrainment in three-dimensional jets is probably equally valid for the full non-linear jet problem and in fact some preliminary experiments have tended to confirm this.

The density anomaly $s$ seen from figure $6(d)$ to be positive above the jet downstream is negative below and hence the downstream jet is likely to be quite unstable. As in the two-dimensional case this is not so upstream since the sign of the anomaly is reversed.

Although the results obtained above are for two- and three-dimensional stokeslets it seems reasonable that some of the basic features determined will 
appear in the non-linear jet solution. We would therefore expect two-dimensional jets to have reverse flows and no entrainment, three-dimensional jets to have entrainment but no reverse flows at finite distance. Downstream jets, it seems likely, will be relatively unstable due to the adverse vertical density gradient across the jet, upstream jets should be relatively stable. It is likely that vertical

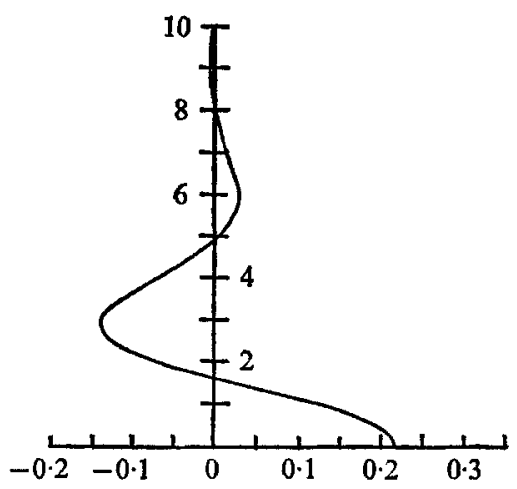

(a)

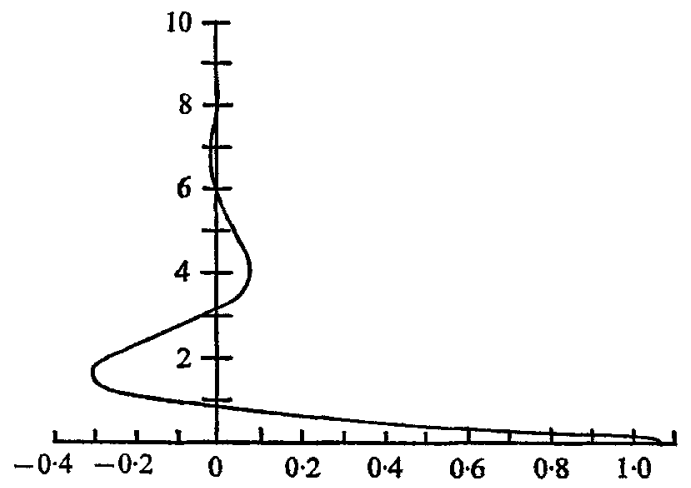

(b)

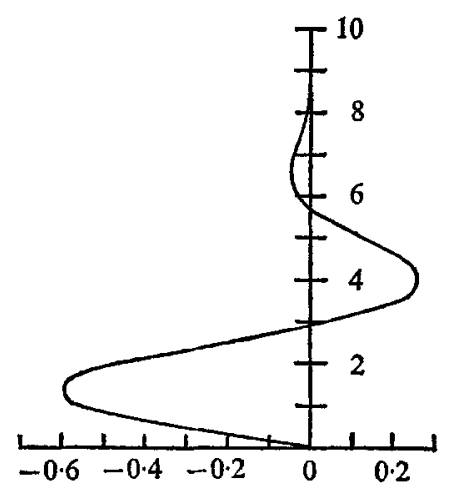

$(c)$

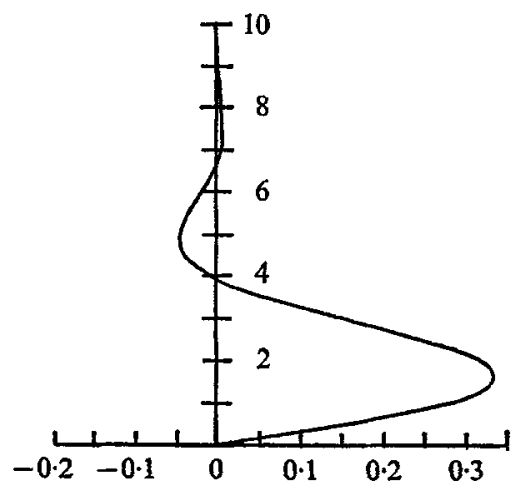

(d)

FIGURE 6. Solutions for the asymptotic diffusive solution functions given in equation (37). (a) $g_{1}(\zeta),(b) g_{2}(\zeta),(c) g_{3}(\zeta),(d) g_{4}(\zeta)$, where $\zeta=|y| / \sigma^{\frac{1}{3}}$.

velocities will be at least an order of magnitude less than horizontal velocities and the basic velocity profiles for the three-dimensional jet will be determined by the solution of the non-diffusive equations of motion with vertical velocity and density anomaly assumed zero. We can, in fact, go further and predict how the velocities will decay.

Consider the $x$ component of the momentum integral for a horizontal jet

$$
X=\int_{S}\left(\sigma_{1 j}-\rho u_{1} u_{j}\right) n_{j} d S
$$

where $S$ is a large surface enclosing the jet and $\sigma_{i j}$ the stress tensor. $S \sim O\left(r^{2}\right)$ so that if the integral is to converge the velocities must be $O\left(r^{-1}\right)$. But if the 
vertical velocity is $O\left(r^{-1}\right)$ then the anomaly term $s$ from (13) must be $O(r)$. But, a consideration of the $y$ momentum integral

$$
-\int_{V} s d V=\int_{S}\left(\sigma_{2 j}-\rho u_{2} u_{j}\right) n_{j} d S
$$

shows that this is not possible.

Thus, we may conclude that the horizontal velocities must decay as $r^{-1}$ but the vertical velocity must be an order of magnitude smaller, in effect, saying that the vertical pressure distribution may be taken as hydrostatic in solving the basic velocity profile. An analysis of the non-linear jet exploiting this idea is in progress.

This research was supported in part by research grant no. 16070 DGY from the Federal Water Pollution Control Administration, U.S. Department of the Interior. The work was initiated while the author was at the Department of Theoretical and Applied Mechanics, University of Auckland, N.Z.

\section{REFERENCES}

HaNCOCK, G.J. 1953 The self-propulsion of microscopic organisms through liquids. Proc. Roy. Soc. A 217, 96-121.

JANowitz, G. S. 1968 On wakes in stratified fluids. $J$. Fluid Mech. 33, 417-432.

LaNDau, L. D. 1944 A new exact solution of the Navier-Stokes equations. Dokl. Ak. Nauk, S.S.S.R. 43, 286-288.

Lovg, R. R. 1959 The motion of fluids with density stratification. J. Geophys. Res. 64, 2151-2163.

LoNG, R. R. 1962 Velocity concentrations in stratified fluids. J. Hyd. Div. A.S.C.E. 88, 9-25.

Longman, I. M. 1956 Note on a method for computing infinite integrals of oscillatory functions. Proc. Camb. Phil. Soc. 52, 764-768.

MORTON, B. R., TAXLOR, G. I. \& TURNER, J. S. 1956 Turbulent gravitational convection from maintained and instantaneous sources. Proc. Roy. Soc. A 236, 1-23.

Schutchting, H. 1933 Laminare Strahlausbreitung. ZAMM, 32, 97-117.

Stezkrn, N. A. 1934 On an exact solution of the oquations of viscous flow. Uch. zap. MGU Sci. Rec., Mascow State Univ. no. 2.

Squrre, H. B. 1951 The round laminar jet. Quart. J. Mech. and Appl. Math. 4, 321-329. 